\title{
Intensity Normalization of Sidescan Sonar Imagery
}

\author{
MS Al-Rawi ${ }^{1}$, Adrián Galdrán ${ }^{2}$, Xin Yuan ${ }^{3}$, Martina Eckert ${ }^{3}$, José-Fernán Martínez ${ }^{3}$, Fredrik Elmgren ${ }^{4}$, Baran \\ Cürüklü ${ }^{5}$, Jonathan Rodriguez ${ }^{1}$, Joaquim Bastos ${ }^{6}$, Marc Pinto ${ }^{7}$ \\ ${ }^{1}$ Departamento de Eletrónica, Telecomunicações e Informática (DETI), Universidade de Aveiro, Aveiro 3810-193, Portugal \\ e-mail: jonathan@ua.pt, al-rawi@ua.pt \\ ${ }^{2}$ Tecnalia Research \& Innovation, Derio, Spain \\ e-mail: agaldran@gmail.com \\ ${ }^{3}$ Research Center on Software Technologies and Multimedia Systems for Sustainability (CITSEM), Universidad Politécnica de Madrid, Spain \\ e-mail: xin.yuan@upm.es, martina.eckert@upm.es,jf.martinez@upm.es \\ ${ }^{4}$ DeepVision AB, Linköping, Sweden \\ e-mail: fredrik@deepvision.se \\ ${ }^{5}$ Mälardalen University, Box 883, 72123 Västerås, Sweden \\ e-mail: baran.curuklu@mdh.se \\ ${ }^{6}$ Instituto de Telecomunicações - Pólo de Aveiro, Aveiro 3810-193, Portugal \\ e-mail: jbastos@av.it.pt \\ ${ }^{7}$ ECA Robotics, 262 rue des frères Lumière, 83130 La Garde, France \\ e-mail: mpi@eca.fr
}

\begin{abstract}
Sonar imaging is currently the exemplary choice used in underwater imaging. However, since sound signals are absorbed by water, an image acquired by a sonar will have gradient illumination; thus, underwater maps will be difficult to process. In this work, we investigated this phenomenon with the objective to propose methods to normalize the images with regard to illumination. We propose to use MIxed exponential Regression Analysis (MIRA) estimated from each image that requires normalization. Two sidescan sonars have been used to capture the seabed in Lake Vättern in Sweden in two opposite directions westeast and east-west; hence, the task is extremely difficult due to differences in the acoustic shadows. Using the structural similarity index, we performed similarity analyses between corresponding regions extracted from the sonar images. Results showed that MIRA has superior normalization performance. This work has been carried out as part of the SWARMs project (http://www.swarms.eu/).
\end{abstract}

Keywords-Sidscan sonar, echo decay, illumination normalization, inverse square law, acoustic shadow, dark channel prior, exponential regression.

\section{INTRODUCTION}

Exploring the seabed is a vital operation that has great importance for industrial, military, and environmental applications. Given the fact that the seas cover $72 \%$ of the surface of the planet Earth and are home to $90 \%$ of all life found on it, underwater imaging has become an active research area in recent years. One essential and distinct part of exploring the seas is imaging the seabed. Here, two main aims are usually considered for underwater imaging: seabed mapping and object recognition/identification with special interest in objects lying on the seabed. This work described here is part of an ambitious project called SWARMS [www.swarms.eu] that aims at making autonomous underwater vehicles (AUVs) and remotely operated vehicles (ROVs) further accessible and useful for autonomous maritime operations, thus providing a viable option for new and existent industries.

Due to the high absorption of electromagnetic waves by water, sonar is the prime imaging device used underwater [1]. Unfortunately, because of the imaging geometry and the way sonar imaging works, acquiring one image of some area of the seabed may not depict the seabed topology correctly. One of the major problems related to underwater sonar imaging is that the acoustic shadow depends on the direction of the sonar head relative to the seabed objects/points-ofinterest [1]. With regard to underwater mapping, maps fusion approaches have been proposed as a viable solution to overcome the weaknesses of using only one map [2,3]. Maps fusion is an extremely complex task that requires sophisticated planning and prudent analysis; where different maps of the seabed may be acquired by different sonars, or using the same sonar but scanning is performed at different directions, and the objective would be fusing all these maps into one entity.

Contrary to the simple description aforementioned, the task is exceptionally challenging due to the harsh underwater environment that imposes problems in image acquisition by sonars. One major problem is the echo decay due to the high absorption rate of sound by water [1]. These factors affect the received echo and thus its strength will depend on the distance the sound has travelled. It is known that the echo decay follow the inverse square law for spherical sound waves $[1,4]$. The consequences of the echo decay on the image quality is a gradient of intensity values ranging from brighter values (seabed points close to the sonar head) that are gradually reduced to the outer borders of the image (seabed points far from the sonar head) [5]. Few models have been proposed to handle the echo decay, for example [4, 5]. The models are based on some hypothesis adapted/modified from the inverse square law or experimental paradigms as the inverse square law may be perturbed due to the environmental factors and the used imaging sonar.

To overcome the weaknesses found in previous approaches, we propose an intensity normalization model using mixed exponential regression analysis (MIRA). The parameters of MIRA are estimated from the image that requires intensity normalization. Another normalization method to sonar imaging was implemented using an adaptation of a well-known fog removal technique, known as Dark Channel Prior Method (DCP).

\section{DATA AND METHODS}

Two sidescan sonars have been simultaneously used to capture the seabed in Lake Vättern in Sweden. The scanning missions have been performed in two different directions, west-east (WE) and east-west (EW), thus a difference of $180^{\circ}$ between the two overlapping regions. To test MIRA and DCP methods, a benchmark have been manually extracted (by the first and the third author) from the non-normalized images (originals) in the form of pairs containing templates extracted from the overlapping regions in WE and EW.

\section{A. Data}

The sonar data was recorded by DeepVision in Lake Vättern in Sweden, an area that is relatively constant in depth. The images have 
been recorded with a DeepEye 680D sidescan sonar (Chirp sonar, $680 \mathrm{kHz}$ ). This sonar is also available in an AUV mounted version that works with the same quality. Two sonars have been used, a left sidescan sonar and a right sidescan sonar. Most of the area is recorded in two directions WE (west east) and (east west) EW, with an overlapping region between $\mathrm{WE}$ and $\mathrm{EW}$. The way the acquisition has been performed is illustrated in Fig. 1. The purpose of this dataset is to provide data for image processing to be used in the context of the SWARMs project in order to prepare maps fusion methods and a toolbox that will be used in the demonstrations and the use cases. Combining the left and right sonar images, each sonar image has more than 5000 pings with each ping having 2000 samples. The acquired data have three WE images and two EW images; for illustration WE3 is shown in Fig. 2, and the outline of the amplitude of one single ping is depicted in Fig. 3.

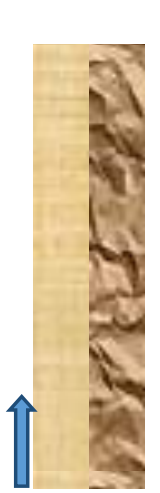

LS RS

WE3

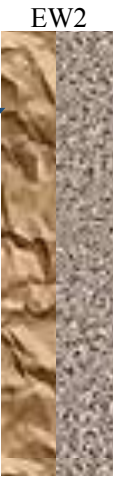

RS LS

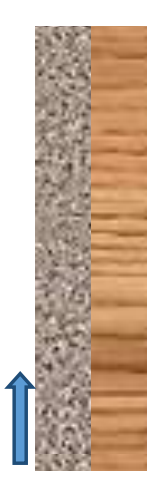

LS RS

WE2

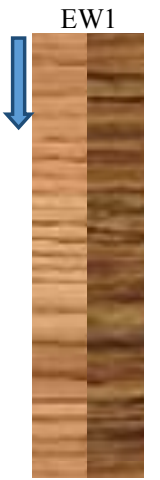

RS LS

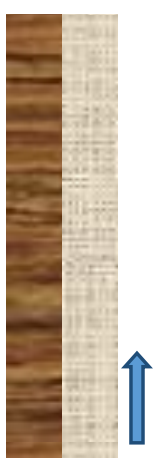

LS RS

Fig. 1. The scanning paradigm used to record the seabed images in Lake Vättern, Sweden. Overlapping regions are marked with a similar canvas pattern. Colors have no meaning here but visual clarity. The arrow exemplifies the movement of the vehicle carrying the imaging sonar.

To test the similarity of the overlapping regions between WE and EW, sub-images of size $400 \times 400$ have been extracted from each of them, and a couple of sub-images representing the same area/region is called a pair. To that end, 19 pairs have been extracted by the first author from the non-normalized images and 13 pairs have been extracted by the third author from the images normalized by MIRA. Afterwards, a template matching method has been implemented to find the corresponding pair in the normalized images (using the 19 pairs) and the non-normalized images (using the 13 pairs).

Similarly, template matching has been implemented to extract 32 pairs from the images normalized by DCP. Due to the difficulty of extracting these pairs from the sonar images, human observer error is highly probable, therefore we tossed few pairs from the benchmark after extraction. To give an example, the extraction of few pairs from overlapping regions is illustrated in Fig. 4, and four pairs extracted from different overlapping regions, along with the effect of intensity normalization, are illustrated in Fig. 5.

Although imaging was performed at roughly the same distance between the sonars and the seabed, image scaling effects were obvious while manually extracting the overlapping regions, not to mention the possible error due to the manual extraction of each pair from the overlapping images which was done by the first and the third authors of this work.

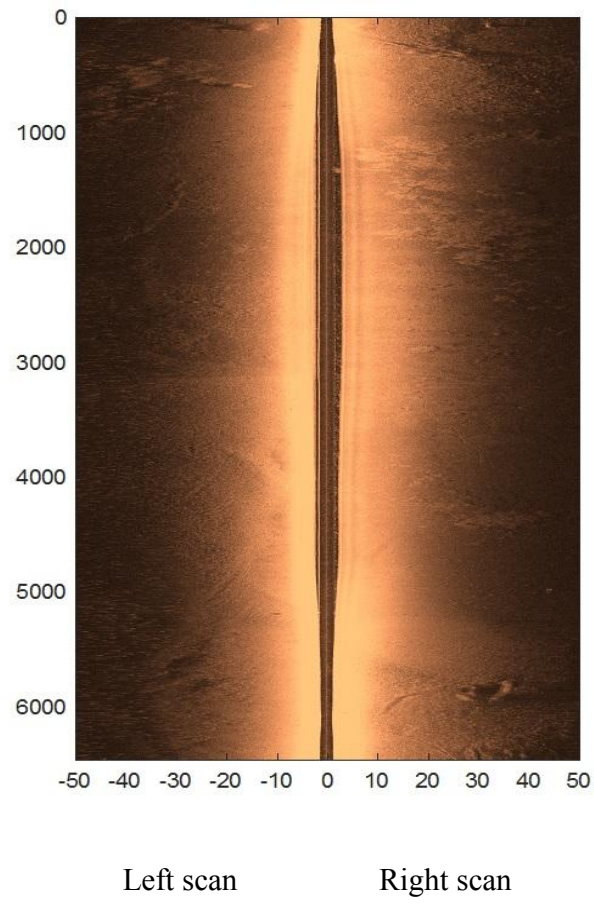

Fig. 2. One original image (WE3) of the seabed displayed without further processing. The vertical axis shows the total number of pings, and the horizontal axis shows the number of samples (1000 in the left, and 1000 in the right), although -50 to 50 is the area covered in meters. $\log (x)$ has been used to compress the received acoustic signal $x$.

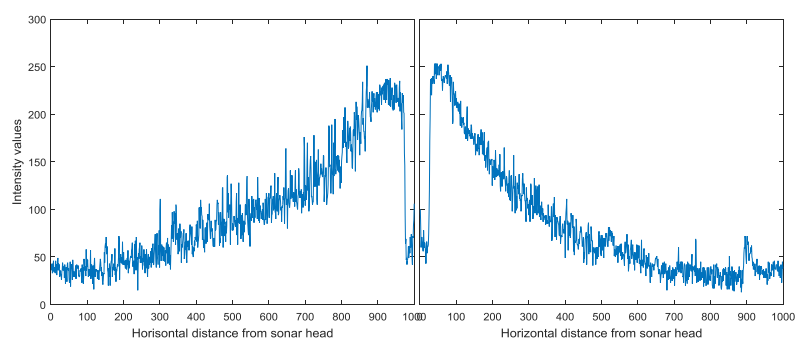

Fig. 3. One ping showing some form of exponential decay in the received acoustic signal, where $\log (x)$ has been used to compress the original values.

\section{B. Methods}

Preprocessing is the very first necessary step in any computer vision and/or image processing task. There are various sources of noise and artefacts in sonar images that need to be removed using specialized filters and methods. One serious artifact that often occurs in sonar imaging is the existence of different intensities in the form of gradient. In sonar imaging, there is a decay in the received echoes as a function of range, due to sound absorption in seawater. This phenomenon is expressed as a non-linear form $f(\mathcal{A}, r)$, where $\mathcal{A}$ is a vector of parameters representing the absorption coefficient depending on sonar frequency, water temperature, depth, etc. and $r$ is the range/distance between the sonar and the object. There is also spherical spreading, expressed as $40 \log (r)$ in the same $\log$ scale. In addition, the seabed backscatter strength varies strongly with grazing angle, hence range and typical variations are in excess of $30 \mathrm{~dB}$. Because of all this total dynamic range requirements of sonars exceed $100 \mathrm{~dB}$. This dynamic range is much too large to be pictured in a single image, thus all sonar manufacturers should implement a proprietary dynamic range 
compression with the objective of reducing the dynamic range to around $40 \mathrm{~dB}$. The goal is not only to make the image "nice to look at", i.e. subjectively, but also to enable using computer vision and image analysis techniques on those corrected/normalized images. In all the images used in this study, $\log (x)$ was used to compress the received acoustic signal $x$.

With regard to the dataset obtained in this study, the images show clearly this deficiency in relation to the range that the acoustic signals have traveled. To demonstrate this, the image in Fig. 2 illustrates that the central region, along the vertical axis, which represents the water column where the left and right signals are combined to form the image, has brighter appearance (higher intensity values). In the same time, the ping at both the left and right boarders appears darker. To illustrate this phenomenon further, one ping is shown in Fig. 3 and it is obvious that the relation between the echo strength and the distance is non-linear, in fact the relation appear to have some form of an exponential model. Therefore, the intensity drift with respect to the closest and the farthest points (in the sonar image) has some form of exponential decay. One way to normalize/correct the intensities is by using regression analysis.

1) Exponential modelling: One way to resolve the illumination change due to the decay in the acoustic signal is by normalizing every pixel in the image according to an estimated weight value. Using exponential regression analysis, the weights can be estimated from the intensity characteristics of the image by treating each ping separately, in our case these are the intensity values of one row in the image, i.e. the received echo of one ping. The simplest exponential model that one can assume is the following:

$$
f(z)=a e^{b z},
$$

where $z$ is the index value at one sample in the ping, $a$ and $b$ are the parameters that should be estimated and $f$ is the output of the fitting model that should be compared to the observed intensity $I(z)$ (via exponential regression analysis). The normalized image should then be estimated using,

$$
\hat{f}(z)=\frac{I(z)}{f(z)} .
$$

For the entire ping, however, we may rewrite (1) as follows:

$$
f(\mathbf{z})=a e^{b z},
$$

where now $z$ is the ping of either the left or the right sidescan sonar, $a$ and $b$ are the weights that can be estimated from all the samples in the ping via regression analysis. While this model might seem sound and might work well, our preliminary experiments showed that it did not fit well to the observed intensity values. As an alternative, we find out that using mixed exponential regression analysis (MIRA) has superior performance, which can be written as follows:

$$
f(\mathbf{z})=a e^{b \mathbf{z}}+\boldsymbol{c} e^{d \mathbf{z}},
$$

where $a, b, c, d$ are now the four weights representing the echo decay for each ping signal, and $\boldsymbol{z}$ is the spatial location (or index) of each sample within the ping. Normalizing the each ping should be done using,

$$
\hat{f}\left(\mathbf{z}_{i}\right)=\frac{I\left(\mathbf{z}_{i}\right)}{f\left(\mathbf{z}_{k}\right)} .
$$

where $k$ is the index of one ping from the image that will be used to find the parameters of MIRA, which could be arbitrary selected or by using a more dedicated selection criteria, $i=1, \ldots, n$, and $n$ is the total number of pings in the image.
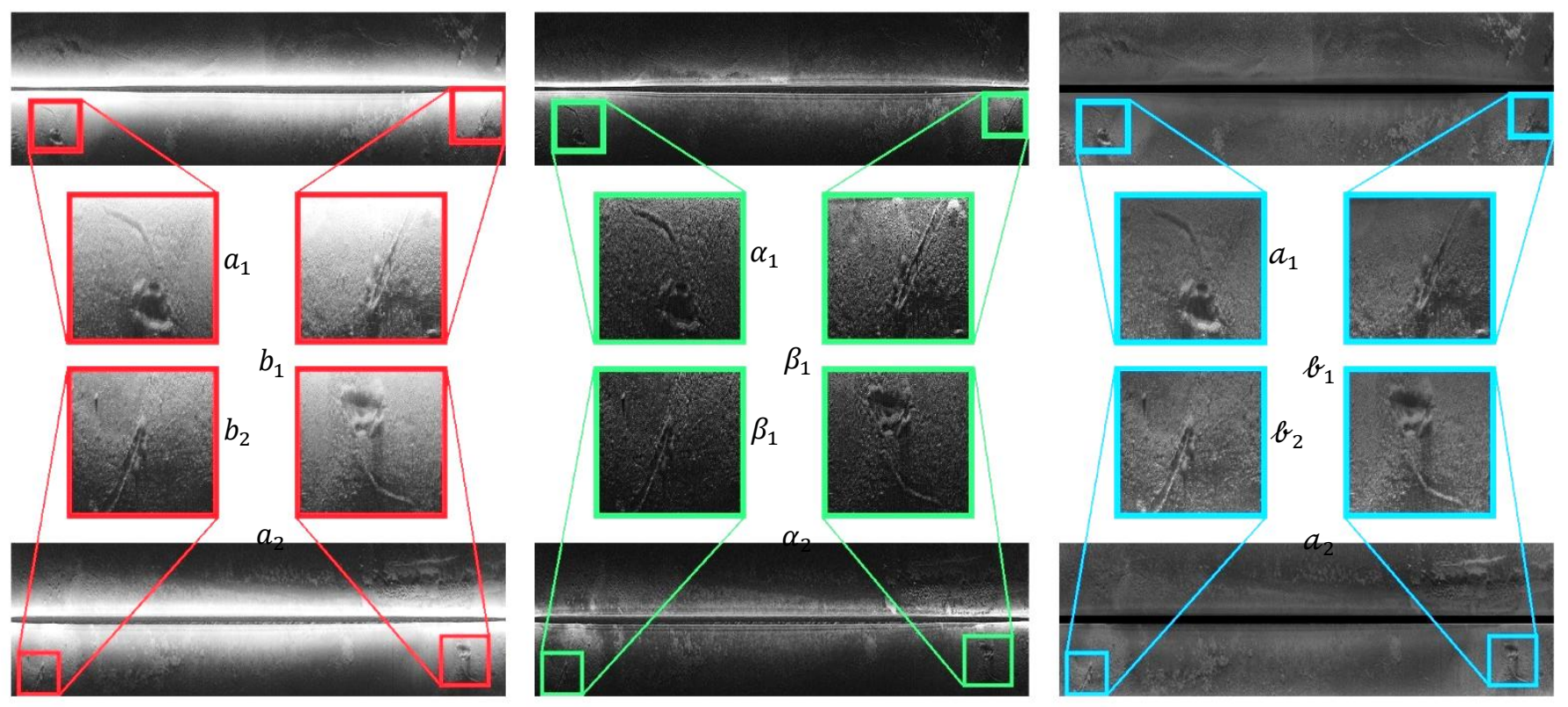

Fig. 4. Extraction of corresponding pairs from, the original image (left), image intensity normalized with the DCP Method (middle), and image intensity normalized with MIRA method (right). We see how the inhomogeneous echo decay produces an irregular illumination field in regions containing natural seabaed landmarks, left part. This artefact seems to be decreased after applying the proposed methods, although the DCP method introduces a considerable amount of artificial contrast in the extracted regions. The shown above are corresponding pairs of distinguished landmarks; 1) original images we have two pairs $\left.\left\{\left(a_{1}, a_{2}\right),\left(b_{1}, b_{2}\right)\right\}, 2\right)$ for DCP we have the two pairs $\left\{\left(\alpha_{1}, \alpha_{2}\right),\left(\beta_{1}, \beta_{2}\right)\right\}$, and 3) for MIRA we have the two pairs $\left\{\left(a_{1}, a_{2}\right),\left(b_{1}, b_{2}\right)\right\}$. 
Non-normalized images
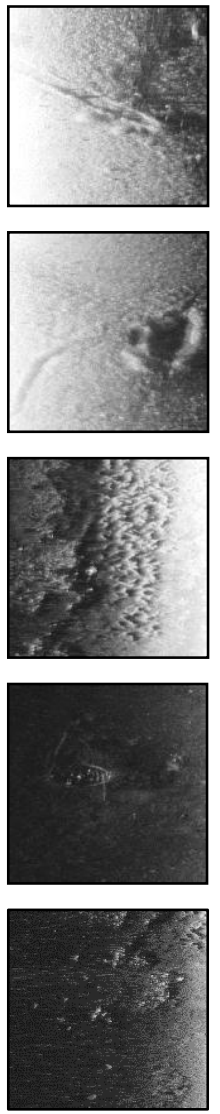
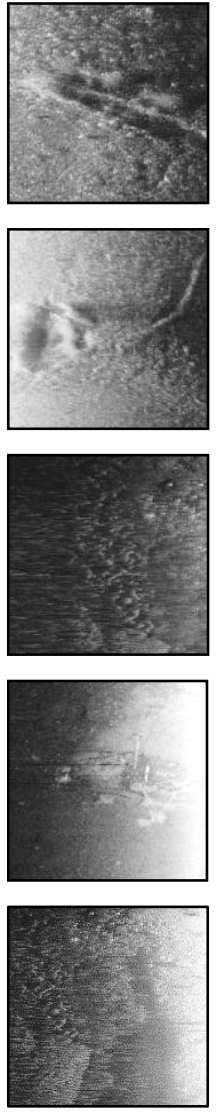

Imges normalized by DCP method
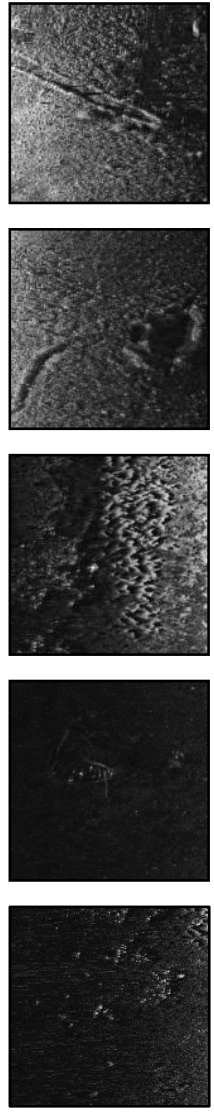
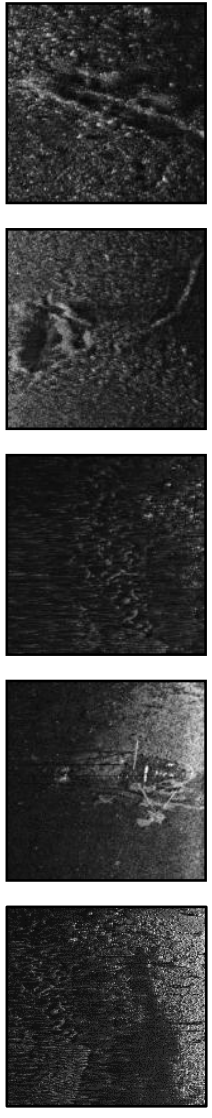

Images normalzied by

MIRA method
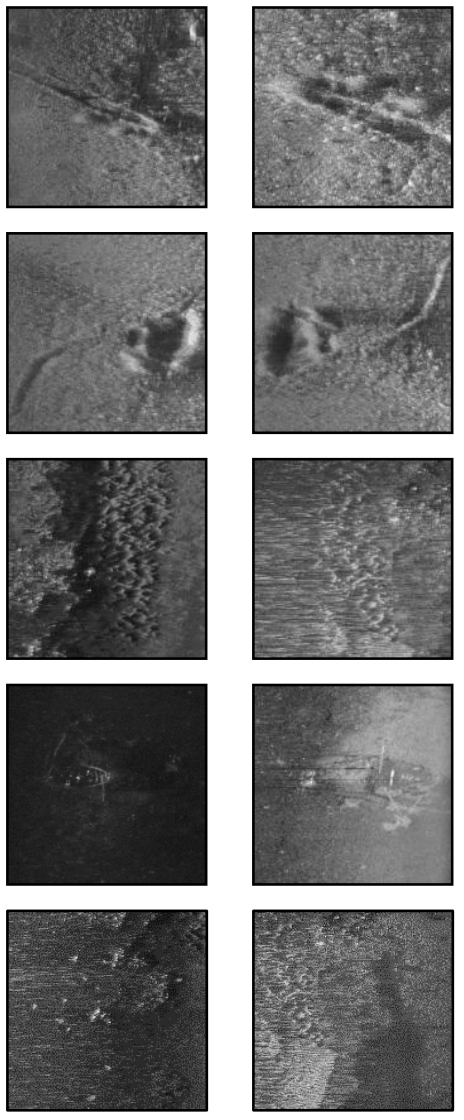

Fig. 5. Four of the 32 pairs contained in the benchmark set. Distinctive landmarks from the original sidescan sonar images, same landmarks after illumination correction by the DCP method and by the MIRA method are shown. It is obvious that some images are difficult to recognize even for the human observer. For illustration purpose, the brightness and contrast of the images shown in this figure have been set to $35 \%$, and the picture boarder of each image was set to black.

Estimating this model for each ping is not only time consuming, but may lead to overfitting. Furthermore, the exponential regression model should be estimated from each of the left and the right images using at least one ping, respectively. The selection of the ping that can be used in the exponential regression analysis is also a problem, since it will be used to normalize all the pings in the image. Nonetheless, our analysis showed that choosing any ping from the image will do, and this case can be used safely if there are not enormous depth changes among the different pings. In this work, we opted to use an arbitrary ping index, at $k=500$. This strategy keeps the computational complexity within the order $O(N)$, where $N$ is the total number of pixels in the image. Additional work, however, might be performed in the future to probably enhance the method further.

For illustration purpose, one ping, at $k=500$, regression analysis and signal normalization are depicted in Fig. 6, where the left MIRA model coefficients (with 95\% confidence bounds) are, $a=229.4$ (210.5, 248.3), $b=-0.005883(-0.006692,-0.005074), c=81.63$ (57.32, 105.9), $d=-0.001001(-0.001335,-0.0006677)$. Right MIRA model coefficients (with $95 \%$ confidence bounds) are, $a=$ $275.2(270.6,279.9), \quad a=-0.00262 \quad(-0.002793, \quad-0.002448)$, $c=0.7158(-1.999,3.431), d=0.002802(-0.0009682,0.006572)$.

2) Dark channel prior method (DCP): The Dark channel method [6] was initially designed to perform image dehazing. The task of image dehazing consists of removing fog from outdoor degraded images [7, 8]. The Dark Channel method attempts to solve the following physical model of atmospheric degradation:

$$
I(x)=J(x) t(x)+A(1-t(x)),
$$

where $I(x)$ is the observed intensity, $J(x)$ is the scene radiance, which corresponds to the non-degraded image, $A$ is a constant additive term usually called airlight, and $t(x)$ is a scalar quantity called transmission, that describes the amount of light that is not scattered nor absorbed, and reaches the observer. According to the BeerLambert law, transmission is exponentially decreasing with respect to the distance, and can be modelled as $t(x)=\exp ^{-\beta(x)}$, where $d(x)$ denotes the distance and $\beta$ is the attenuation coefficient of the medium the signal traverses.

To invert the above model and retrieve $J(x)$, one needs to impose some constraints on the problem. The Dark Channel method imposes that no large bright areas without contrast should be present in the retrieved radiance, see [6] for the technical details. In this sense, although in principle image dehazing and echo decay compensation seem to be unrelated problems, both of them share a common feature: their goal is to produce contrast and shrink intensities in too bright areas of the image, while keeping intensity unaltered in dark areas. As shadows contain useful information in sonar scans, and the Dark Channel method will leave those areas unmodified, it seems to be a reasonable solution to test in order to solve the echo decay mitigation problem. 
3) Local similarity analysis: In order to assess the performance of the two proposed methods, we analyze the similarity of the landmarks extracted from different scans of the same area after correction of the echo decay.

In a first stage, we employ the well-known Structural Similarity Index (SSIM) [9] to evaluate the similarity between a landmark and its $180^{\circ}$-rotated counterpart, see Fig. 4 . The SSIM measures the similarity of two images in terms of three independent variables: luminance, contrast and structure. We define the mean and variance of a signal $\boldsymbol{x}$ (in this case containing several pixels) of size $N$ as:

$$
\mu_{x}=\frac{1}{N} \sum_{i=1}^{N} x_{i} ; \sigma_{x}=\frac{1}{N-1}\left(\sum_{i=1}^{N}\left(x_{i}-\mu_{x}\right)^{2}\right)^{\frac{1}{2}},
$$

and the covariance between two signals $x$ and $y$ as:

$$
\sigma_{x y}=\frac{1}{N-1} \sum_{i=1}^{N}\left(x_{i}-\mu_{x}\right)\left(y_{i}-\mu_{y}\right) .
$$

Last, we define a comparison function as follows:

$$
L(a, b)=\frac{2 a b}{a^{2}+b^{2}} .
$$

Then we have that the SSIM index is the product of a luminance comparison, a contrast comparison, and a structure comparison:

$$
\operatorname{SSIM}(x, y)=L\left(\mu_{x}, \mu_{y}\right) L\left(\sigma_{x}, \sigma_{y}\right) s(x, y),
$$

where $s(x, y)=\frac{\sigma_{x y}}{\sigma_{x} \sigma_{y}}$.

i)

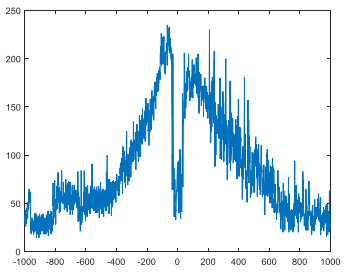

iii)

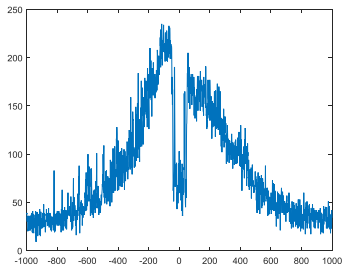

iv)

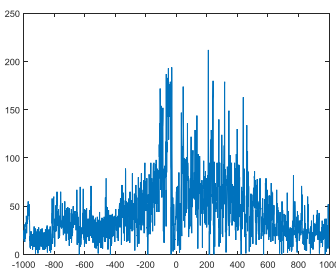

vi)

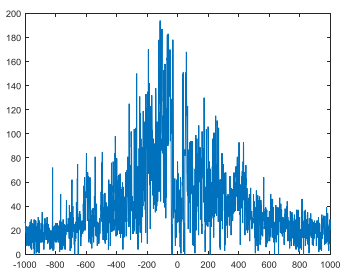

ii)
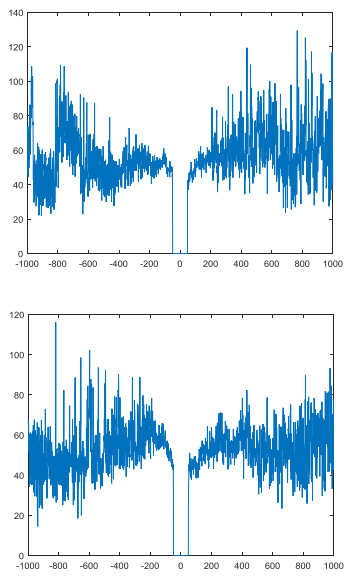

Fig. 6. Using image WE3. i) Ping at index 500 used to estimate the MIRA model; where two models are estimated one for the left scan/ping and another for the right scan/ping, ii) ping 500 after normalizing it with the estimated MIRA model, iii) another ping from the same image at index 4000, iv) after normalizing ping 4000 with the same MIRA model estimated from ping 500, v) ping 500 normalized with DCP, and vi) ping 4000 normalized with DCP.
4) Global quality analysis: Apart from the above local analysis, we may also perform a global analysis (considering the entire sonar image instead of sub-regions as in Fig. 4) of the effect of applying the proposed techniques. While the above local analyses are useful to assess the feasibility of a subsequent landmark matching stage after correcting the initial seabed scans, a global analysis may be useful for several tasks, such as providing a mechanism to automatically tune the parameters of an echo decay correction algorithm, or evaluating its correctness without the need to manually extract natural landmarks.

To perform such a global analysis, we propose to study the difference of echo values between adjacent regions of the sonar scan. To that end, we divide the sonar image into a collection of $M \times N$ patches covering the whole scanned seabed. Then, for each patch $(m, n)$ we compute its mean intensity, which we denote by $\mu_{m n}$. Afterwards, to estimate the quality of an image patch, we compare its mean intensity with the adjacent ones following Weber's perceptual law:

$$
C_{m, n}=\frac{\max \left(\mu_{m, n}-\mu_{m_{k}, n_{k}}\right)}{\mu_{m, n}} .
$$

Finally, we define a metric, that we call SIQEM (Sonar Image Quality Assessment Metric), as follows:

$$
\operatorname{SIQEM}(S)=\frac{1}{M} \frac{1}{N} \sum_{m=1}^{M} \sum_{n=1}^{N} C_{m, n}
$$

The lower SIQEM value an image attains, the better quality it has. A similar image quality metric has been recently proposed in [10] for uneven illumination assessment purposes in dermoscopic images of the skin, but to the best of our knowledge, no similar quality metric has been proposed before to measure echo decay in side-scan sonar scans of the seabed.

\section{EXPERIMENTAL RESULTS}

We computed the SSIM value of the 19 different landmark pairs present in our dataset. The mean and standard deviation of the SSIM score obtained by both normalization methods, and also without applying any normalization, are shown in Table I.

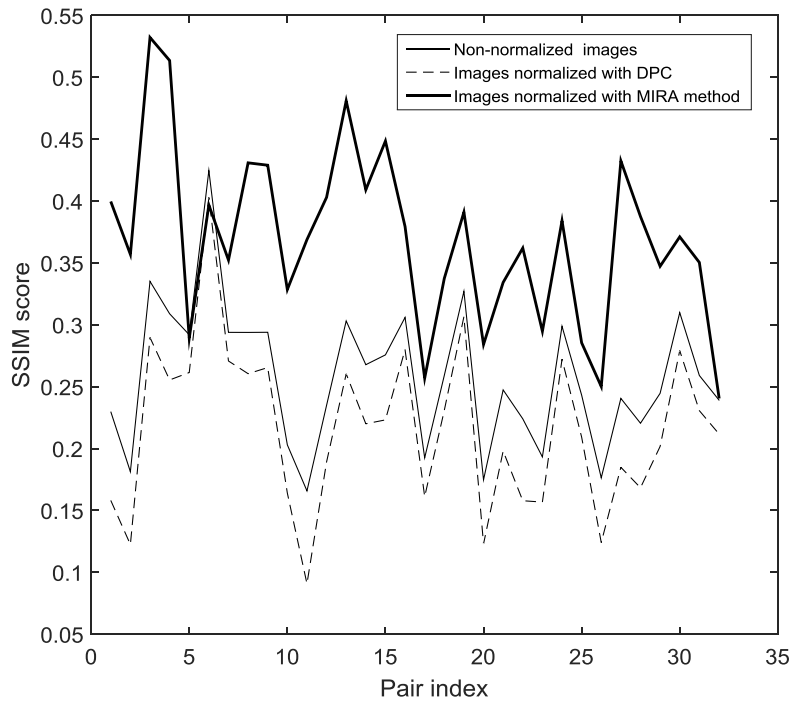

Fig. 7. SSIM scores measuring the similarity of the two methods proposed in this work and compared to the non-normalized images. MIRA (the dark thick line) outperforms the non-normalized images and the DCP. 
TABLE I. MEAN AND STANDARD DEVIATION OF SSIM SCORES ON THE ENTIRE SET OF LANDMARK PAIRS AVAILABLE IN THIS STUDY

\begin{tabular}{ccc}
\hline Non-normalized & DCP & MIRA \\
\hline $0.258 \pm 0.057$ & $0.217 \pm 0.066$ & $0.369 \pm 0.071$ \\
\hline
\end{tabular}

We see that the MIRA method performs the best at correlating different landmarks coming from different sidescan sonars, while the DCP method seems to reduce the mean value of the SSIM score for most of the available landmarks pairs and it even lower than the nonnormalized images.

Regarding the global quality evaluation, the values of the SIQEM metric for the five sonar scans available in our dataset, after processing with both enhancement methods and without any processing, are shown in Table II.

TABLE II. RESULTS OF THE SIQEM METRIC ON EACH OF THE FIVE SIDE SCAN SONARS IN THE USED DATASET, THE LOWER SIQEM THE BETTER IMAGE QUALITY

\begin{tabular}{cccc}
\hline $\begin{array}{c}\text { Image set } \\
\text { extracted from }\end{array}$ & $\begin{array}{c}\text { Non- } \\
\text { normalized }\end{array}$ & DCP & MIRA \\
\hline WE3 & 0.115 & 0.129 & 0.076 \\
\hline EW2 & 0.120 & 0.143 & 0.087 \\
\hline WE2 & 0.117 & 0.142 & 0.080 \\
\hline EW1 & 0.109 & 0.125 & 0.076 \\
\hline WE1 & 0.137 & 0.157 & 0.107 \\
\hline Mean \pm STD & $0.12 \pm 0.01$ & $0.14 \pm 0.01$ & $0.08 \pm 0.01$ \\
\hline
\end{tabular}

We see how in every case that the MIRA method outperforms the DCP, the latter seems to perform worse than the non-normalized images. These results are in line with the above local analysis and validate the value of the SIQEM metric for globally evaluating the behavior of illumination normalization that has been verified subjectively.

\section{CONCLUSIONS}

This study has shown that handling the echo decay in images acquired by sidescan sonars by means of exponential modelling is effective. Such preprocessing techniques will lead to improved representation and classification of underwater images and thus will facilitate maps fusing, matching, registration, among other objectives. The manual extraction of the pairs from the overlapping regions in two sonar images acquired via opposite direction missions has been a difficult task. This is due to the severe differences in the acoustic shadows between the overlapping regions in the images. Thus, the cases where the similarity scores of few images normalized with the MIRA method have been comparable to the scores of the non-normalized images could be related to the imperfect pair extraction. The possible translation, slight-rotation, and scale differences (or even an affine transform between every pair) that has been provoked during the movement of the vehicle carrying the two sidescan sonars could be another reason of this occasional low performance. Hence, deploying an affine invariant metric to measure the similarity between every pair may result in greater improvement. Although the current MIRA model only estimates its parameters from one ping, local and global similarity analysis indicated its effectiveness in solving the echo decay problem.

Further work to optimize the exponential model, for example by including more pings in the regression analysis, might lead to improving MIRA's performance even more, although such modelling will have to be compromised with the computational complexity and the available computational resources. It will also be a worthy notion to use statistical analysis techniques, such as permutation testing [11], to support further the validity, generalization, and reliability of the MIRA method. From the results and the benchmark prepared in this study, the MIRA method still needs further tuning and parameter optimization in order to produce better normalized images.

\section{ACKNOWLEDGMENT}

The research leading to the presented results has been undertaken within the SWARMs European project (Smart and Networking Underwater Robots in Cooperation Meshes), under Grant Agreement n. 662107-SWARMs-ECSEL-2014-1, which is partially supported by the ECSEL JU and the Fundação para a Ciência e a Tecnologia (FCT; ECSEL/0003/2014), the Spanish National Plan for Scientific Tech. Res. and Innov: TEC2013-48453-C2-2-R, and China Scholarship Council (CSC).

\section{REFERENCES}

[1] R. P. Hodges, Underwater Acoustics: Analysis, Design and Performance of Sonar: Wiley, 2010.

[2] A. Burguera, and G. Oliver, "High-Resolution Underwater Mapping Using Side-Scan Sonar," Plos One, vol. 11, no. 1, Jan, 2016.

[3] A. Nait-Chabane, B. Zerr, and G. Le Chenadec, "Rangeindependent segmentation of sidescan sonar images," Traitement Du Signal, vol. 30, no. 3-5, pp. 119-148, May-Oct, 2013.

[4] Y.-C. Chang, S.-K. Hsu, and C.-H. Tsai, "Sidescan Sonar Image Processing: Correcting Brightness Variation and Patching Gaps," Journal of Marine Science and Technology-Taiwan, vol. 18, no. 6, pp. 785-789, Dec, 2010.

[5] C. G. Capus, A. C. Banks, E. Coiras, I. T. Ruiz, C. J. Smith, and Y. R. Petillot, "Data correction for visualisation and classification of sidescan SONAR imagery," IET Radar Sonar and Navigation, vol. 2, no. 3, pp. 155-169, Jun, 2008.

[6] K. He, J. Sun, and X. Tang, "Single Image Haze Removal Using Dark Channel Prior," IEEE Transactions on Pattern Analysis and Machine Intelligence, vol. 33, no. 12, pp. 2341-2353, Dec, 2011.

[7] J.-P. Tarel, and N. Hautiere, "Fast Visibility Restoration from a Single Color or Gray Level Image," IEEE International Conference on Computer Vision. pp. 2201-2208, 2009.

[8] A. Galdran, J. Vazquez-Corral, D. Pardo, and M. Bertalmio, "Enhanced Variational Image Dehazing," Siam Journal on Imaging Sciences, vol. 8, no. 3, pp. 1519-1546, 2015, 2015.

[9] Z. Wang, A. C. Bovik, H. R. Sheikh, and E. P. Simoncelli, "Image quality assessment: From error visibility to structural similarity," IEEE Transactions on Image Processing, vol. 13, no. 4, pp. 600612, Apr, 2004.

[10] Y. Lu, F. Xie, Y. Wu, Z. Jiang, and R. Meng, "No Reference Uneven Illumination Assessment for Dermoscopy Images," IEEE Signal Processing Letters, vol. 22, no. 5, pp. 534-538, May, 2015.

[11] M. S. Al-Rawi, and J. P. S. Cunha, "On using permutation tests to estimate the classification significance of functional magnetic resonance imaging data," Neurocomputing, vol. 82, pp. 224-233, Apr, 2012. 